\title{
Handgrip Strength Predicts Difficult Weaning But Not Extubation Failure in Mechanically Ventilated Subjects
}

\author{
Guillaume Cottereau PT, Martin Dres MD, Alexandre Avenel MD, Jérome Fichet MD, \\ Frédéric M Jacobs MD, Dominique Prat MD, Olfa Hamzaoui MD, Christian Richard MD PhD, \\ Marc Antonello PT, and Benjamin Sztrymf MD PhD
}

\begin{abstract}
BACKGROUND: Muscle weakness, defined by the Medical Research Council scale, has been associated with delay in mechanical ventilation weaning. In this study, we evaluated handgrip strength as a prediction tool in weaning outcome. METHODS: This was a 1-y prospective study in 2 ICUs in 2 university hospitals. Adult patients who were on mechanical ventilation for at least $48 \mathrm{~h}$ and eligible for mechanical ventilation weaning were screened for inclusion. Handgrip strength was evaluated using a handheld dynamometer before each spontaneous breathing trial (SBT). Attending physicians were unaware of handgrip strength and decided on extubation according to guidelines. RESULTS: Eighty-four subjects were included (median age 66 [53-79] y, with a median Simplified Acute Physiology Score II of 49 [37-63]). At the first evaluation, median handgrip strength was significantly associated with weaning outcome as defined by international guidelines: simple (20 [12-26] kg), difficult $(12[6-21] \mathrm{kg})$, or prolonged $(6[3-11] \mathrm{kg})$ weaning $(P=.008)$. Time to liberation from mechanical ventilation and ICU stay were significantly longer for subjects classified as having muscle weakness according to the handgrip strength-derived definition $(P=.02$ and $P=.03$, respectively). In multivariate analysis, known history of COPD (odds ratio [OR] 5.48, 95\% CI 1.44-20.86, $P=.01$ ), sex (OR 6.16, 95\% CI 1.64-23.16, $P=.007)$, and handgrip strength at the first SBT (OR 0.89, 95\% CI $0.85-0.97, P=.004)$ were significantly associated with difficult or prolonged weaning. Extubation failure, as defined by re-intubation or unscheduled noninvasive ventilation within $48 \mathrm{~h}$ after extubation, occurred 14 times after 92 attempts, leading to an extubation failure rate of $15 \%$. No association was found between handgrip strength and extubation outcome. CONCLUSIONS: Muscle weakness, assessed by handgrip strength, is associated with difficult or prolonged mechanical ventilation weaning and ICU stay, but not with extubation outcome. Key words: handgrip strength; handheld dynamometry; intensive care unit-acquired weakness; muscle weakness; mechanical ventilation; weaning. [Respir Care 2015;60(8):1097-1104. (C) 2015 Daedalus Enterprises]
\end{abstract}

\section{Introduction}

Patients staying in the ICU frequently develop muscle weakness involving limbs and respiratory muscles. ${ }^{1,2}$ This condition is involved in delayed weaning and/or extubation failure mainly due to respiratory muscle dysfunction and/or inadequate cough. ${ }^{3,4}$ The Medical Research Coun-

\footnotetext{
Supplementary material related to this paper is available at http:// www.rcjournal.com.

Mr Cottereau and Dr Dres are co-first authors.

Dr Sztrymf presented a version of this paper at the 41st Congress of the Société de Réanimation de Langue Française, held January 16, 2013, in Paris, France.
}

The authors have disclosed no conflicts of interest. 


\section{Handgrip Strength Predicts Difficult Weaning}

cil (MRC) scale, a bedside test requiring an experienced physiotherapist, is considered the accepted standard for evaluation of peripheral muscle strength. ${ }^{5}$ This test was used in previous studies demonstrating the negative impact of muscle weakness on mechanical ventilation weaning. 3,6 There is growing interest in dominant-handgrip

See the Related Editorial on Page 1213

strength for evaluation of muscle strength. It can be monitored quickly, noninvasively, and very easily with a handheld dynamometer. ${ }^{7}$ Although monitoring only the dominant-hand maximum pressure delivered, handgrip strength was reported as significantly correlated to the MRC scale score and appears to be a reliable alternative. ${ }^{8}$ For this purpose, a handgrip-based definition of acquired weakness has been proposed for critical care patients. ${ }^{8}$ To our knowledge, this tool has never been tested in the setting of mechanical ventilation weaning. Therefore, the main goal of this study was to evaluate handgrip strength as a predictor of weaning duration. We also sought to test the value of handgrip strength in predicting extubation outcome.

\section{Methods}

\section{Design and Settings}

This prospective study was conducted over a 12-month period (May 1, 2011 to May 1, 2012) in 2 ICUs (10 and 15 beds, respectively) from the same institution (Assistance Publique-Hôpitaux de Paris, Hôpitaux Universitaires ParisSud, Université Paris 11, France). The protocol was approved by the ethics committee of the Société de Réanimation de Langue Française (agreement 11-325). All subjects gave informed consent to participate.

\section{Subjects}

Adult patients ( $>18 \mathrm{y}$ of age) were eligible for enrollment if they required mechanical ventilation for at least $48 \mathrm{~h}$ and if they met the predefined ready-to-wean criteria on the daily screening (see study protocol). Patients unable to perform the test were excluded (any rheumatologic conditions, previously known abnormal limitations of strength, amputations, muscular disease, cachexia state). Neuropsy-

Correspondence: Benjamin Sztrymf MD PhD, Réanimation Médicale, Hôpital Antoine-Béclère, 157 Rue de la Porte de Trivaux, 92140 Clamart, France. E-mail: benjamin.sztrymf@abc.aphp.fr.

DOI: $10.4187 /$ respcare.03604

\section{QUICK LOOK}

\section{Current knowledge}

Patients in the ICU frequently develop muscle weakness involving limbs and respiratory muscles as a result of prolonged immobility. This muscle weakness may be involved in delayed weaning and/or extubation failure, owing to respiratory muscle dysfunction and/or inadequate cough.

\section{What this paper contributes to our knowledge}

Muscle weakness, assessed by handgrip strength, is associated with difficult or prolonged mechanical ventilation weaning and ICU stay, but not with extubation outcome.

chiatric symptoms, such as confusion and delirium, as assessed with the Confusion Assessment Method for the Intensive Care Unit ${ }^{9}$ at the time of enrollment were also a reason for exclusion.

\section{Handgrip-Strength Evaluation}

Trained physiotherapists assessed handgrip strength. Subjects were positioned as close to upright as possible, with the shoulders in neutral rotation at the subject's side and elbow flexion of $90^{\circ}$. An adjustable handheld dynamometer (Jamar hydraulic hand dynamometer, Fred Sammons, Bolingbrook, Illinois) was used to obtain handgripstrength measurements. The dominant hand of each subject was tested. As recommended by the manufacturer, subjects were asked to hold the dynamometer at their maximum strength for 2-3 s. Examiners also demonstrated the test and encouraged subjects. The highest handgrip strength after 3 consecutive attempts, separated by brief pauses ( $\sim 30 \mathrm{~s})$, was monitored.

Muscle weakness was diagnosed on the basis of the previously published handgrip strength-derived definition of ICU-acquired weakness $(<11 \mathrm{~kg}$ for men and $<7 \mathrm{~kg}$ for women). ${ }^{7}$ Because only a normalized comparison can confirm whether there are differences in handgrip strength, the handgrip value is expressed as an absolute value in kilograms and as a relative value as a percentage, calculated according to Bohannon et $\mathrm{al},{ }^{10}$ with the values of healthy subjects taking into account sex and age. We also normalized the value of handgrip strength according to body height because of the known impact of this anthropometric characteristic on handgrip strength. ${ }^{11}$

\section{Study Protocol}

In both investigating centers, weaning was conducted according to international guidelines (see the supplemen- 


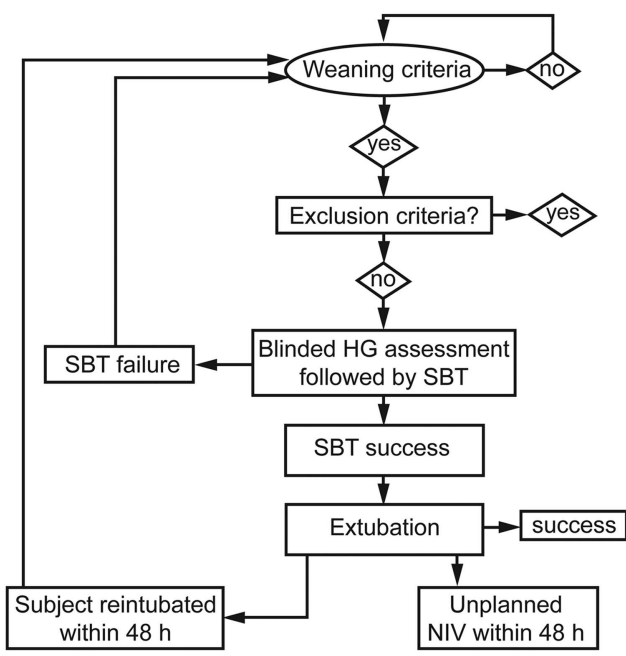

Fig. 1. Study protocol. $H G=$ handgrip, $S B T=$ spontaneous breathing trial, NIV = noninvasive ventilation.

tary materials at http://www.rcjournal.com). ${ }^{12}$ Once daily, eligibility for ventilator weaning was evaluated according to international guidelines (Fig. 1). Handgrip strength of the dominant hand was evaluated by the attending physiotherapist just before each spontaneous breathing trial (SBT). The attending physician was unaware of the result of the test. In the case of SBT failure, reasons for failure were explored, and another SBT was performed as soon as possible. In the case of SBT success, the attending physician, made the decision to extubate, relying only on the SBT and clinical patterns according to international guidelines. In case of re-intubation, another handgrip-strength evaluation was performed following the weaning trial. Daily physiotherapy management, feeding, and sedation policies are described in the supplementary materials.

\section{Data}

Demographic data (sex, age, dominant hand, body mass index, comorbidities) were recorded at admission. We also registered the reason for mechanical ventilation, Simplified Acute Physiology Score II (SAPS II) at admission, duration of mechanical ventilation until each SBT, handgrip strength, intravenous sedative drug administration, caloric intake, use of neuromuscular blocking agents and steroids and their cumulative doses, and time from first SBT to extubation. The reasons for extubation failure were explored according to the recommended diagnosis framework. ${ }^{10}$

\section{Statistical Analysis}

On the basis of published data in the field, we expected simple weaning to occur in $50-60 \%$ of subjects without

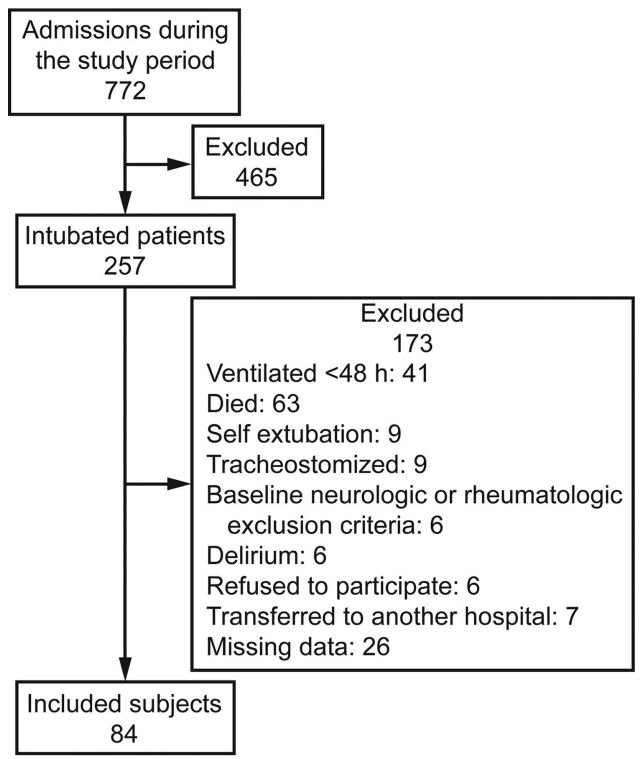

Fig. 2. Flowchart of the study.

muscle weakness ${ }^{13}$ and difficult or prolonged weaning in $60 \%$ of subjects with muscle weakness. ${ }^{14}$ Therefore, we estimated that 86 examined subjects would provide $80 \%$ power to detect this difference with a 2 -sided a level of .05 .

StatView 5.0 (SAS Institute, Cary, North Carolina) was used to perform statistical analysis. Data are expressed as median (interquartile range). Continuous variables were compared using the Mann-Whitney $U$ test or the KruskalWallis test when comparing at least 3 groups. Categorical variables were compared using the chi-square test or the Fisher exact test as appropriate. To identify factors associated with difficult weaning, we selected variables by univariate analysis $(P<.1)$ and entered them in a stepwise logistic regression analysis. $P \leq .05$ was considered statistically significant. The receiver operating characteristic curve, featuring 1 -specificity on the $\mathrm{X}$ axis and sensitivity on the $\mathrm{Y}$ axis for the different values, was calculated. The area under the receiver operating characteristic curve and best absolute handgrip-strength threshold were determined.

\section{Results}

\section{Population}

During the study period, 722 subjects were admitted. Among them, 257 underwent mechanical ventilation, and 84 were included in the study. The reasons for exclusion are shown in Figure 2. The median age was 66 (53-79) y, and the median SAPS II score was 49 (37-63). The reasons for admission and baseline data are reported in Table 1. The median duration of mechanical ventilation before 
Table 1. Characteristics of the 84 Subjects

\begin{tabular}{|c|c|}
\hline Characteristic & Values \\
\hline Age, y & $66(53-79)$ \\
\hline Females/males, $n$ & $45 / 39$ \\
\hline COPD, $n(\%)$ & $25(31)$ \\
\hline Chronic heart failure, $n(\%)$ & $20(24)$ \\
\hline SAPS II & $49(37-63)$ \\
\hline BMI, $\mathrm{kg} / \mathrm{m}^{2}$ & $25(21-30)$ \\
\hline Dominant hand (right/left), $n$ & $75 / 9$ \\
\hline \multicolumn{2}{|l|}{ Indications for ICU admission, $n$} \\
\hline Acute respiratory failure & 34 \\
\hline Shock & 24 \\
\hline Coma & 9 \\
\hline Seizures & 3 \\
\hline Intoxication & 10 \\
\hline Thrombotic thrombocytopenic purpura & 1 \\
\hline Cardiac arrest & 1 \\
\hline Rhabdomyolysis & 1 \\
\hline Gastrointestinal bleeding & 1 \\
\hline Sepsis, $n(\%)$ & $58(68.2)$ \\
\hline $\begin{array}{l}\text { Mechanical ventilation duration before } \\
\text { first weaning trial, } d\end{array}$ & $6(3-14)$ \\
\hline Mechanical ventilation duration, $\mathrm{d}$ & $9(4-17)$ \\
\hline $\begin{array}{l}\text { Continuous intravenous sedation } \\
\text { duration before first weaning trial, } \mathrm{d}\end{array}$ & $3(2-7)$ \\
\hline Steroid treatment, $n(\%)$ & $17(20)$ \\
\hline Cumulative amount of steroids, mg* & $280(115-548)$ \\
\hline $\begin{array}{l}\text { Neuromuscular blocking agent treatment, } \\
n(\%)\end{array}$ & $18(21)$ \\
\hline Cumulative amount of atracurium, mg & $100(50-998)$ \\
\hline Daily caloric intake, $\mathrm{kcal} / \mathrm{kg} / \mathrm{d}$ & $18(13-23)$ \\
\hline ICU stay, d & $13(7-24)$ \\
\hline Handgrip at first SBT, kg & $15(7-23)$ \\
\hline $\begin{array}{l}\text { Results are expressed as median (interquartile range) un } \\
\text { * Results are expressed in prednisone equivalent dose. } \\
\text { SAPS II = Simplified Acute Physiology Score II } \\
\text { BMI=body mass index } \\
\text { SBT = spontaneous breathing trial }\end{array}$ & erwise. \\
\hline
\end{tabular}

the first SBT was 6 (3-14) d. All but 13 subjects received continuous intravenous sedation. Six subjects died during the study period.

\section{Subject Characteristics According to SBT}

Simple, difficult, and prolonged weaning occurred in 41 (49\%), 33 (39\%), and 10 (12\%) subjects, respectively. The subjects in these subgroups differed according to age, underlying COPD, mechanical ventilation duration, ICU stay, and handgrip strength at the first SBT (Table 2 and Fig. $3 \mathrm{~A})$. We tested the difference in absolute values of handgrip strength normalized according to an age-matched and sex-matched population and found no significant difference between subgroups (Table 2 and Fig. 3B). The percentage of subjects receiving steroids, neuromuscular blocking agents, and vasopressors was not different between these subgroups (see the supplementary materials at http://www.rcjournal.com).

\section{Handgrip-Strength Evaluation}

The first median handgrip strength was 15 (7-23) kg, which represents $29 \%$ (25-41\%) of the theoretical strength of a sex-matched and age-matched healthy population. ${ }^{10}$ Muscle weakness was diagnosed in $31 \%$ of our cohort.

\section{Risks Factors of Difficult or Prolonged Weaning and ICU Stay}

Time to liberation from mechanical ventilation was significantly longer for subjects with muscle weakness as defined by the handgrip strength-derived definition $(P=.02)$ (Fig. 4A). In univariate analysis, age, sex, known history of COPD, handgrip strength at the first SBT, and use of continuous intravenous sedation were significantly associated with difficult or prolonged weaning. In multivariate analysis, known history of COPD (odds ratio [OR] $5.48,95 \%$ CI $1.44-20.86, P=.01$ ), $\operatorname{sex}($ OR $6.16,95 \%$ CI 1.64-23.16, $P=.007)$, and handgrip strength at the first SBT (OR $0.89,95 \%$ CI $0.85-0.97, P=.004$ ) were significantly associated with difficult or prolonged weaning (Table 3). A receiver operating characteristic analysis was performed to test the acuity of handgrip strength in predicting weaning outcome. The area under the curve was 0.66 . Handgrip strength had 0.76 sensitivity and 0.69 specificity for identifying difficult or prolonged weaning at a cutoff of $11 \mathrm{~kg}$ (see the supplementary materials at http:// www.rcjournal.com). Subjects with muscle weakness according to the handgrip strength-derived definition were more likely to have a longer ICU stay $(P=.03)$ (Fig. 4B).

Re-intubation or unscheduled noninvasive ventilation within $48 \mathrm{~h}$ after extubation occurred 14 times after 92 attempts, leading to an extubation failure rate of $15 \%$. The median handgrip strength at the first extubation attempt for each subject was not different according to extubation outcome (16 [7-23] kg for extubation success vs 10 [5-18] $\mathrm{kg}$ for extubation failure, $P=.14$ ). Prophylactic noninvasive ventilation was used in 9 subjects. Tracheotomy was performed in 2 subjects. The reasons for extubation failure were laryngeal edema $(n=4)$, aspirations $(n=3)$, cardiogenic pulmonary edema $(n=1)$, atelectasis $(n=1)$, and other causes of acute respiratory failure $(n=5)$.

\section{Discussion}

Our findings can be summarized as follows. (1) Muscle weakness as defined by low handgrip strength was associated with difficult or prolonged weaning. (2) No association between handgrip strength and extubation outcome 
Table 2. Subject Characteristics According to Weaning Outcome

\begin{tabular}{|c|c|c|c|c|}
\hline Characteristic & Simple $(n=41)$ & Difficult $(n=33)$ & Prolonged $(n=10)$ & $P^{*}$ \\
\hline Age, y & $58(55-74) \dagger$ & $68(58-79)$ & $69(67-79) \ddagger$ & .03 \\
\hline Females/males, $n$ & $26 / 15 \dagger$ & $13 / 20$ & $6 / 4$ & .11 \\
\hline Height, $\mathrm{m}$ & $1.69(1.61-1.75)$ & $1.73(1.67-1.76)$ & $1.65(1.53-1.70)$ & .09 \\
\hline Weight, $\mathrm{kg}$ & $69(62.1-88.9)$ & $76.2(63.6-95.5)$ & $67.5(55.3-80.5)$ & .36 \\
\hline SAPS II & $47(35-55)$ & $52(38-63)$ & $60(39-80)$ & .18 \\
\hline Known history of COPD, $n(\%)$ & $7(17)$ & $13(39.4)$ & $5(50) \ddagger$ & .05 \\
\hline Baseline chronic heart failure, $n(\%)$ & $8(19.5)$ & $7(21.2)$ & $5(50)$ & .14 \\
\hline Mechanical ventilation duration, $\mathrm{d}$ & $4(3-12) \dagger$ & $11(8-17)$ & $20(17-22) \ddagger$ & .001 \\
\hline ICU stay, d & $8(5-16) \dagger$ & $17(12-26)$ & $24(20-31) \ddagger$ & $<.001$ \\
\hline $\begin{array}{l}\text { Mechanical ventilation duration before first } \\
\text { weaning trial, } d\end{array}$ & $4(3-11)$ & $8(4-14)$ & $12(11-16)$ & .16 \\
\hline \multicolumn{5}{|l|}{ Handgrip at first SBT } \\
\hline $\mathrm{kg}$ & $20(12-26) \dagger$ & $12(6-21) \S$ & $6(3-11) \div$ & .008 \\
\hline$\% \|$ & $30(23-42)$ & $29(27-41)$ & $25(22-34)$ & .53 \\
\hline $\mathrm{kg} / \mathrm{m} \mathbb{I}$ & $11(8-15) \dagger$ & $6(4-12) \S$ & $3(2-6) \ddagger$ & .003 \\
\hline Muscular weakness, $n(\%)^{* *}$ & $6(15) \dagger$ & $13(39)$ & $5(50) \ddagger$ & .006 \\
\hline \multicolumn{5}{|c|}{$\begin{array}{l}\text { Results are expressed as median (interquartile range) unless indicated otherwise. } \\
\text { SAPS II = Simplified Acute Physiology Score II } \\
\text { SBT }=\text { spontaneous breathing trial } \\
\text { * Comparison of the } 3 \text { subgroups with Kruskal-Wallis test. } \\
\dagger P<.05 \text { when comparing simple and difficult groups (Mann-Whitney or Fisher exact test). } \\
\ddagger P<.05 \text { when comparing simple and prolonged groups (Mann-Whitney or Fisher exact test). } \\
\S P<.05 \text { when comparing difficult and prolonged groups (Mann-Whitney or Fisher exact test). } \\
\| \text { Percentage of an age-matched and sex-matched healthy population. } \\
\text { I Handgrip strength in kg normalized by body height in m. } \\
* * \text { Muscular weakness according to handgrip-based definition. }\end{array}$} \\
\hline
\end{tabular}
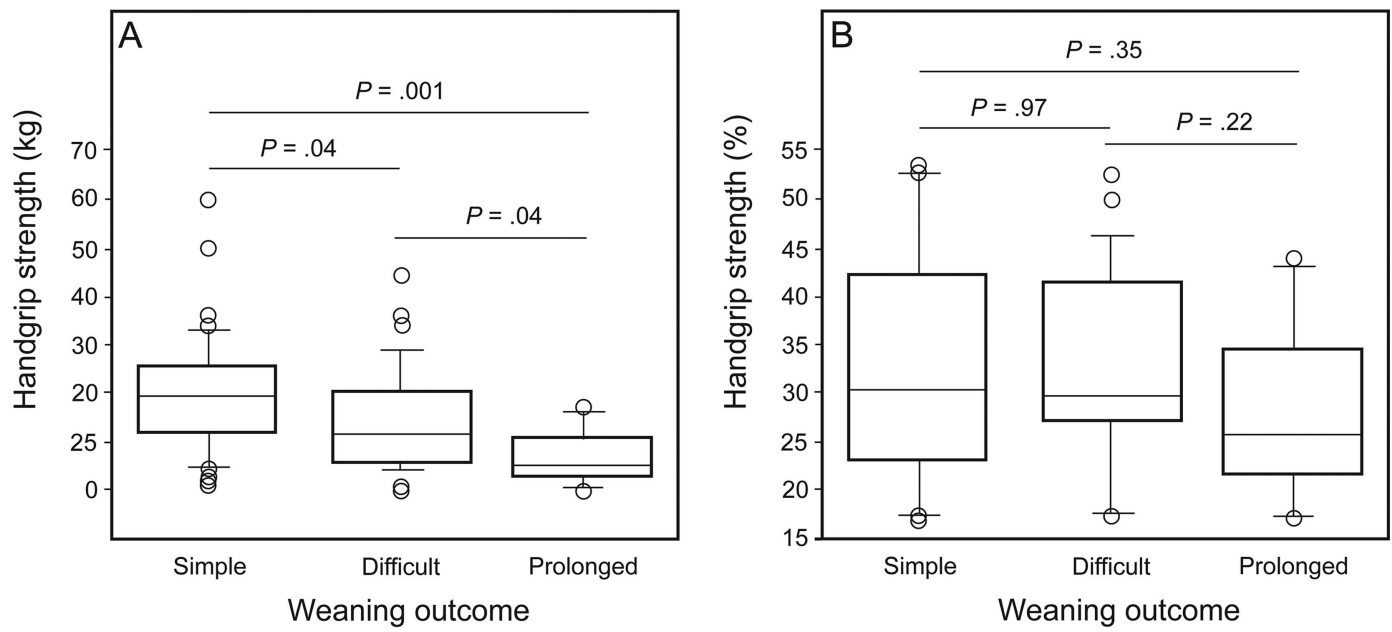

Fig. 3. Handgrip strength according to weaning outcome. A: Handgrip strength of subjects at the first spontaneous breathing trial (SBT). B: Absolute values of handgrip strength in age-matched and sex-matched healthy controls at the first SBT. Box plots represent the 1st and 3rd quartiles; the horizontal line at the center is the median. Whiskers denote highest and lowest values within 1.5 interquartile of the highest and lowest quartiles. Dots show outliers. A: $P=.008$. B: $P=.53$ with the Kruskal-Wallis test.

was found. (3) The values of the first handgrip-strength evaluation were associated with ICU stay.

Our results show that low handgrip strength evaluated before initiating the weaning process was weakly but independently associated with difficult or prolonged weaning. Our results were confirmed by normalization of ab- solute values of handgrip strength to body height. This finding supports the relevance of this reliable and simple tool in the prediction of prolonged or difficult weaning, although it must be noted that the area under the curve of 0.66 points indicated a moderate discriminatory value. Because it has been stated that weaning failure occurs be- 

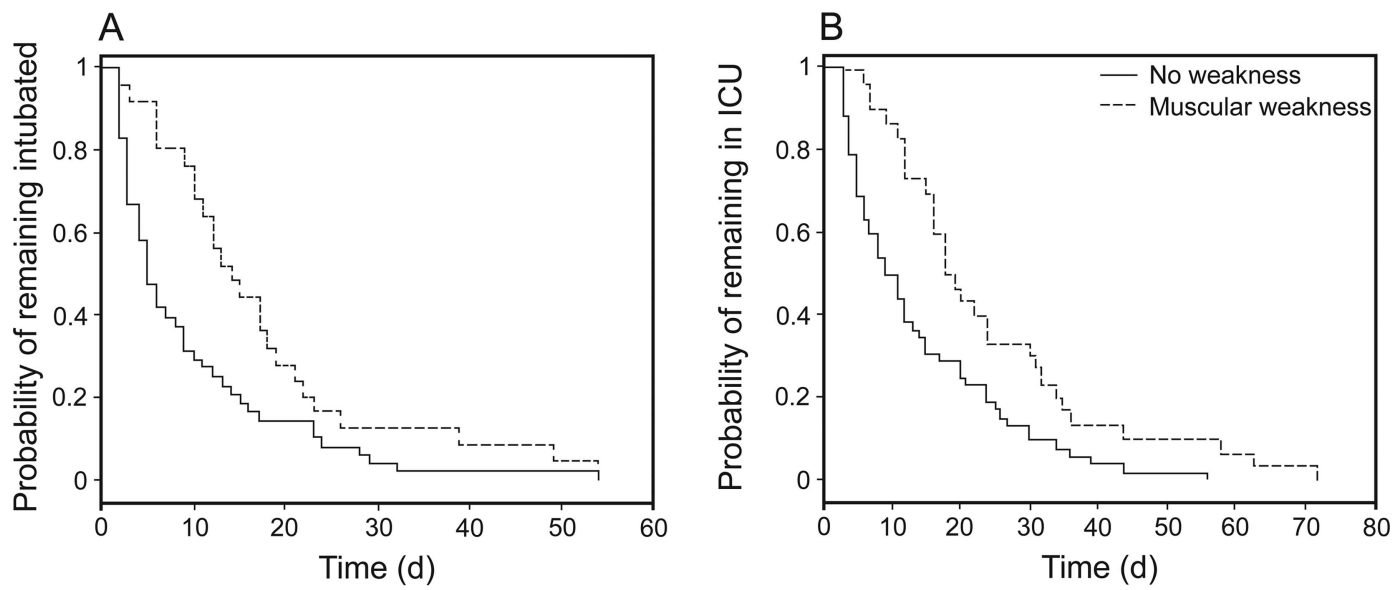

Fig. 4. Kaplan-Meier curves of probability of remaining intubated $(A)$ and in the ICU (B) according to muscle weakness status. $A: P=.02$. $\mathrm{B}: P=.03$ with the log-rank test.

Table 3. Factors Associated With Difficult or Prolonged Weaning

\begin{tabular}{|c|c|c|c|c|c|c|}
\hline & \multicolumn{3}{|c|}{ Univariate Analysis } & \multicolumn{3}{|c|}{ Multivariate Analysis } \\
\hline & OR & $95 \% \mathrm{CI}$ & $P$ & OR & $(95 \%) \mathrm{CI}$ & $P$ \\
\hline Age & 1.04 & $1.01-1.07$ & .01 & 1.03 & $0.99-1.06$ & .22 \\
\hline Height & 1.02 & $0.98-1.07$ & .33 & & & \\
\hline Weight & 1.01 & $0.98-1.03$ & .69 & & & \\
\hline Gender & 2.54 & $1.06-6.12$ & .038 & 6.16 & $1.64-23.16$ & .007 \\
\hline Known history of COPD & 3.24 & $1.18-8.89$ & .02 & 5.48 & $1.44-20.86$ & .01 \\
\hline Handgrip strength & 0.95 & $0.91-0.99$ & .02 & 0.89 & $0.85-0.97$ & .004 \\
\hline Continuous intravenous sedation & 4.67 & $1.18-18.42$ & .03 & 3.0 & $0.61-14.75$ & .18 \\
\hline $\begin{array}{l}\text { Mechanical ventilation duration before first } \\
\text { weaning trial }\end{array}$ & 1.03 & $0.98-1.07$ & .23 & & & \\
\hline
\end{tabular}

cause of imbalance between respiratory muscle capacity and respiratory load, ${ }^{15}$ several explanations for this relationship can be proposed. Evaluation of the peripheral group of muscles (ie, dominant hand) could reflect evaluation of the whole muscle activity. ${ }^{7}$ Moreover, handgrip strength correlates with strength of other muscle groups, ${ }^{16}$ although limits have been suggested in precisely characterizing overall strength with this tool. ${ }^{17}$ This is explained by the influence of multiple muscular and non-muscular factors on the handgrip test, such as anthropometric characteristics, psychological status, disease severity, inflammation, oxidative stress, physical activity, comorbidities, medications, and environmental factors. ${ }^{18}$ In this regard, it has been suggested that handgrip strength be considered as a global functional physical test rather than a simple muscle test. This might explain the relationship between this test and weaning outcome in our study. Indeed, although muscle strength has been associated with diaphragmatic strength in mechanically ventilated patients, ${ }^{19}$ handgrip strength might indicate a more global performance status, thus representing a determinant point in the setting of mechanical ventilation weaning. In addition, it has been shown that patients with chronic respiratory and/or cardiac diseases have lower exercise capacity and lower handgrip strength compared with a matched population without these disabilities. ${ }^{20}$

Anticipating the consequences of muscle weakness in extubation outcome remains a daily challenge in the ICU. Sometimes, muscle weakness, as clinically estimated, appears to be the ultimate obstacle for patients who succeed in a weaning trial. Among these patients, it is very difficult to predict who will exhibit post-extubation respiratory failure and require re-intubation, which carry a pejorative prognosis. $^{21}$ Our results do not suggest that performing handgrip-strength evaluation could help to predict the failure of extubation and of weaning from mechanical ventilation. Several reasons could explain this. First, because several mechanisms are involved in extubation failure, ${ }^{12}$ the evaluation of one factor among many is not sufficient to predict the outcome after extubation. Second, our sam- 


\section{Handgrip Strength Predicts Difficult Weaning}

ple size was small and led to a low number of extubation failures. Nevertheless, our extubation failure rate is in the same range as mean aggregate data from interventional or observational studies $(15.7 \pm 15.6 \%$ and $15.0 \pm 14.0 \%$, respectively). ${ }^{22}$ Furthermore, in our study, laryngeal edema, which is not related to muscle status, explained $>28 \%$ of the extubation failures $(n=4)$. Aspiration was the cause of extubation failure in $21 \%$ of cases. Although an association between handgrip strength and tongue strength has been evidenced in a cohort of geriatric patients outside the ICU, ${ }^{23}$ the occurrence of laryngeal injury and subsequent swallowing disorders among recently extubated patients has not been directly associated so far with muscle weakness in the general ICU population. ${ }^{24}$ Therefore, according to the aforementioned limitations of extubation outcome analysis, we think that this point deserves to be further investigated in a larger population.

Our study has some limitations. First, we did not measure the MRC scale score and could not correlate it with handgrip-strength evaluation. Nevertheless, because in our units, physiotherapists currently perform both MRC scale score and handgrip-strength evaluations, we wanted to avoid any potential bias. Second, we studied the strength of only one muscle group (ie, the dominant hand). It would be interesting to correlate our findings with evaluation of diaphragmatic function because we might have included subjects with predominant ventilation-induced diaphragmatic dysfunction, which could have blurred the link between handgrip strength and weaning failure. Nevertheless, more invasive investigations would have been required, which we could not perform in the setting of this study. Third, both proximal and distal muscles are usually affected in acquired weakness, and although a significant correlation between MRC scale score and handgrip strength has been reported previously, ${ }^{7}$ it is possible that handgripstrength evaluation does not appropriately explore musclestrength decrease in a subset of patients with predominant proximal disease. Fourth, 9 subjects in our study underwent prophylactic noninvasive ventilation because of an estimated high risk of weaning failure. Due to the potentially important benefit of this management strategy, we determined that it was appropriate and ethical to provide this support to subjects with risk of extubation failure. Whether this influenced the results of our study is unknown, but because our aim was to test handgrip strength in the daily practice of mechanical ventilation weaning, we decided not to exclude these subjects from the statistical analysis. Fifth, the percentage of the theoretical handgrip strength of a sex-matched and age-matched healthy population was not different among the 3 weaning outcome subgroups. This would have been the best way of normalizing absolute handgrip values. We think that this might be related to the low number of subjects, the mathematical transformation of absolute values of handgrip strength, and the influence of other factors influencing both muscle strength and weaning outcome, Last, our handgrip-based definition of muscle weakness was based on the study by Ali et al, ${ }^{7}$ in which subjects were studied from day 5 onward, whereas we studied subjects from day 2 onward. It should also be noted that this study demonstrated a moderate overall sensitivity and specificity $(80.6 \%$ and $83.2 \%$, respectively) compared with the MRC measurement method. This could have biased the muscle status classification of our subjects.

\section{Conclusions}

Handgrip strength, when obtained before the first SBT, is an independent prognostic factor of mechanical ventilation weaning outcome and ICU stay. Our results do not suggest an association between handgrip strength and extubation outcome. Nevertheless, further data are needed to confirm this issue.

\section{ACKNOWLEDGMENTS}

We thank Yen N'Guyen PT, Sandrine Mercier PT, Pedro Pereira PT, Dragos Zus PT, and Juan Robles PT (Physiotherapy and Rehabilitation Department, Hôpital Antoine-Béclère, Clamart, France) and, Gisele Charlot PT, and Ruben Sanchez PT (Physiotherapy and Rehabilitation Department, Hôpital Bicêtre, Le Kremlin-Bicêtre, France) for technical assistance.

\section{REFERENCES}

1. Visser L. Critical illness polyneuropathy and myopathy: clinical features, risk factors and prognosis. Eur J Neurol 2006;13(11):1203-1212.

2. Sharshar T, Bastuji-Garin S, Stevens RD, Durand MC, Malissin I, Rodriguez $\mathrm{P}$, et al. Presence and severity of intensive care unitacquired paresis at time of awakening are associated with increased intensive care unit and hospital mortality. Crit Care Med 2009;37(12): 3047-3053.

3. De Jonghe B, Bastuji-Garin S, Durand MC, Malissin I, Rodrigues P, Cerf C, et al. Respiratory weakness is associated with limb weakness and delayed weaning in critical illness. Crit Care Med 2007;35(9): 2007-2015.

4. Garnacho-Montero J, Amaya-Villar R, García-Garmendía JL, Madrazo-Osuna J, Ortiz-Leyba C. Effect of critical illness polyneuropathy on the withdrawal from mechanical ventilation and the length of stay in septic patients. Crit Care Med 2005;33(2):349-354.

5. Stevens RD, Marshall SA, Cornblath DR, Hoke A, Needham DM, de Jonghe B, et al. A framework for diagnosing and classifying intensive care unit-acquired weakness. Crit Care Med 2009;37(10 Suppl): S299-S308.

6. De Jonghe B, Bastuji-Garin S, Sharshar T, Outin H, Brochard L. Does ICU acquired paresis lengthen weaning from mechanical ventilation. Intensive Care Med 2004;30(6):1117-1121.

7. Ali NA, O'Brien JM Jr, Hoffmann SP, Phillips G, Garland A, Finley JC, et al. Acquired weakness, handgrip strength, and mortality in critically ill patients. Am J Respir Crit Care Med 2008;178(3):261-268.

8. Bittner EA, Martyn JA, George E, Frontera WR, Eikermann M. Measurement of muscle strength in the intensive care unit. Crit Care Med 2009;37(10 Suppl):S321-S330. 


\section{Handgrip Strength Predicts Difficult Weaning}

9. Ely EW, Inouye SK, Bernard GR, Gordon S, Francis J, May L, et al. Delirium in mechanically ventilated patients: validity and reliability of the Confusion Assessment Method for the Intensive Care Unit (CAM-ICU). JAMA 2001;286(21):2703-2710.

10. Bohannon RW, Peolsson A, Massy-Westropp N, Desrosiers J, BearLehman J. Reference values for adult grip strength measured with a Jamar dynamometer: a descriptive meta-analysis. Physiotherapy 2006;92(1):11-15.

11. Spruit MA, Sillen MJ, Groenen MT, Wouters EF, Franssen FM. New normative values for handgrip strength: results from the UK biobank. J Am Med Dir Assoc. 2013;14:775.e5-11.

12. Boles JM, Bion J, Connors A, Herridge M, Marsh B, Melot C, et al. Weaning from mechanical ventilation. Eur Respir J 2007;29(5):10331056.

13. Thille AW, Richard JC, Brochard L. The decision to extubate in the intensive care unit. Am J Respir Crit Care Med 2013;187(12):1294 1302.

14. Perren A, Brochard L. Managing the apparent and hidden difficulties in mechanical ventilation weaning. Intensive Care Med 2013;39(11): 1885-1895.

15. McConville JF, Kress JP. Weaning patients from the ventilator. NEngl J Med 2012;367(23):2233-2239.

16. Samuel D, Rowe P. An investigation of the association between grip strength and hip and knee joint moments in older adults. Arch Gerontol Geriatr 2012;54(2):357-360.
17. Bohannon RW. Is it legitimate to characterize muscle strength using a limited number of measures? J Strength Cond Res 2008;22(1):166-173.

18. Stenholm S, Tiainen K, Rantanen T, Sainio P, Heliövaara M, Impivaara O, Koskinen S. Long-term determinants of muscle strength decline: prospective evidence from the 22-year mini-Finland follow-up survey. J Am Geriatr Soc 2012;60(1):77-85.

19. Tzanis G, Vasileiadis I, Zervakis D, Karatzanos E, Dimopoulos S, Pitsolis T, et al. Maximum inspiratory pressure, a surrogate parameter for the assessment of ICU-acquired weakness. BMC Anesthesiol 2011;11:14

20. Cheung CL, Nguyen US, Au E, Tan KC, Kung AW. Association of handgrip strength with chronic diseases and multimorbidity: a crosssectional study. Age 2013;35(3):929-891

21. Thille AW, Harrois A, Schortgen F, Brun-Buisson C, Brochard L. Outcomes of extubation failure in medical intensive care unit patients. Crit Care Med 2011;39(12):2612-2618.

22. Krinsley JS, Reddy PK, Iqbal A. What is the optimal rate of failed extubation? Crit Care 2012;16(1):111

23. Butler SG, Stuart A, Leng X, Wilhelm E, Rees C, Williamson J, Kritchevsky SB. The relationship of aspiration status with tongue and handgrip strength in healthy older adults. J Gerontol A Biol Sci Med Sci 2011;66(4):452-458.

24. Tadié JM, Behm E, Lecuyer L, Benhmamed R, Hans S, Brasnu D, et al. Post-intubation laryngeal injuries and extubation failure: a fiberoptic endoscopic study. Intensive Care Med 2010;36(6): 991-998.

This article is approved for Continuing Respiratory Care Education credit. For information and to obtain your CRCE

(free to AARC members) visit

www.rcjournal.com

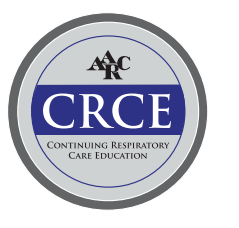

